\title{
EFEITOS ADVERSOS NO PÓS-OPERATÓRIO DE CIRURGIAS GINECOLÓGICAS E MAMÁRIAS
}

\author{
Adriana Cássia Paiva Santos; franklin Sarmento da Silva Braga; Angélica de fátima Assunção Braga; Gustavo Antonio de Souza; Sirlei Siani \\ Morais; Luiz Carlos Zeferino*
}

Trabalho realizado no Centro de Atenção Integral à Saúde da Mulher - UNICAMP, Campinas, SP

\author{
Correspondência: \\ Departamento de \\ Tocoginecologia/CAISM/UNICAMP \\ Rua Alexander Fleming, 101 \\ Cidade Universitária "Zeferino Vaz" \\ CEP: 13083-970, Campinas, SP \\ Fone: (19) 3788-7138 \\ FAX: (19) 3788-8010 \\ zeferino@caism.unicamp.br
}

\begin{abstract}
RESUMO
Oвjetrvos. Analisar a ocorrência de efeitos adversos no período de 24 horas da evolução pós-anestésica e pós-cirúrgica em mulheres submetidas a intervenções cirúrgicas ginecológicas e mamárias de pequeno porte e identificar os principais fatores associados.

Métodos. Trata-se de estudo tipo corte transversal que incluiu 159 mulheres submetidas a intervenções cirúrgicas ginecológicas e mamárias consideradas de pequeno porte. As mulheres foram internadas no dia anterior à intervenção cirúrgica e permaneceram no hospital por pelo menos 24 horas após o término do procedimento. As técnicas anestésicas realizadas foram bloqueio intercostal, bloqueio espinhal e anestesia geral.

Resultados. Os eventos adversos mais freqüentes foram vômitos, náuseas e dor, e ocorreram em 40,3\% das mulheres. Destes, $60 \%$ foram observados nas primeiras quatro horas e $80 \%$ em até seis horas, após a intervenção cirúrgica. As mulheres submetidas ao bloqueio intercostal apresentaram alta mais precoce da recuperação anestésica; o bloqueio espinhal esteve associado mais freqüentemente à dor no período pós-operatório, porém houve menor incidência de náuseas e vômitos em relação à anestesia geral e bloqueio intercostal. Antecedente de tabagismo contribuiu para ocorrência de dor. Conclusão. $O$ período de seis horas de observação mostrou-se adequado para a avaliação da maioria das complicações e efeitos adversos que ocorrem após intervenções cirúrgicas, ginecológicas e mamárias, de pequeno porte.
\end{abstract}

UnITERMOs: Cirurgia ambulatorial. Ginecologia. Mama. Anestesia.

\section{INTRODUÇÃo}

Avanços tecnológicos na área da saúde têm possibilitado a realização de procedimentos cirúrgicos menos invasivos com o objetivo de reduzir incidência de complicações, tempo de internação e custos hospitalares. $\mathrm{O}$ atendimento em nível ambulatorial constitui alternativa à realização de procedimentos cirúrgicos de pequeno porte, sob anestesia geral ou regional, que permitam pronta ou rápida recuperação do paciente, sem necessidade de pernoite, sendo, no entanto, necessário garantir segurança e conforto para a paciente!.

Em geral, os efeitos adversos relatados no pós-operatório imediato de cirurgias de pequeno porte são náuseas e vômitos, hemorragia, dor, cefaléia e febre ${ }^{1-3}$. A freqüência com que estes efeitos ocorrem pode estar associada às condições clínicas pré-operatórias, à extensão e ao tipo de intervenção cirúrgica, às intercorrências cirúrgicas ou anestésicas e à eficácia das medidas terapêuticas adotadas ${ }^{4-11}$. Estudos retrospectivos com grande amostragem não relataram óbito, e um número pequeno de complicações graves foi identificado. Nestes estudos, dor, vômitos e sangramento da ferida operatória constituem as principais causas de reinternação $0^{12,13}$.

Todavia, falta conhecimento ou análise recente de possíveis complicações e efeitos adversos relacionados às intervenções cirúrgicas ginecológicas e mamárias de pequeno porte, que possam servir de referencial na determinação do tempo adequado para a avaliação pósoperatória. Portanto, o objetivo deste estudo foi analisar a ocorrência de efeitos adversos no período de 24 horas da evolução pós-anestésica e pós-cirúrgica em mulheres submetidas a intervenções cirúrgicas ginecológicas e mamárias de pequeno porte e identificar os principais fatores associados.

\section{Métodos}

Trata-se de estudo tipo corte transversal, realizado no período de julho a setembro de 2003. Após aprovação pelo Comitê de Ética do Hospital e assinatura de consentimento livre e esclarecido, foram incluídas 159 mulheres que aceitaram participar deste estudo e assinaram o termo. Elas foram submetidas a intervenções cirúrgicas consideradas de pequeno porte nas regiões pélvica (colo uterino e vulva) e torácica (mama e inserção de Portocath $®$ ), sob anestesia geral, bloqueio espinhal ou bloqueio intercostal. Por se tratar de hospital universitário, com equipes cirúrgica e anestésica fixas por dia da semana, os procedimentos cirúrgicos e anestésicos foram realizados por diferentes equipes. Os procedimentos foram classificados de pequeno porte, segundo critérios adotados pelo serviço e relacionados à extensão e duração do ato operatório.

Todas as mulheres foram internadas no dia anterior à interven- 
ção cirúrgica e permaneceram no hospital por pelo menos 24 horas após o seu término. Os dados obtidos durante a avaliação pré-anestésica e os constantes na ficha de anestesia foram coletados pelo anestesiologista; os relacionados ao procedimento cirúrgico e à recuperação pós-anestésica (RPA) foram informados pelo cirurgião responsável e pela equipe de enfermagem, respectivamente. As complicações após alta da RPA foram diagnosticadas pelo médico responsável e pela equipe de enfermagem que atua na enfermaria. Todas estas informações e dados foram anotados nos prontuários médicos e transcritos para uma ficha pré-codificada, criada para esta finalidade.

$\mathrm{Na}$ avaliação pré-operatória, foi investigada história de tabagismo, presença de comorbidades como hipertensão arterial, diabetes melito e cardiopatia. O tabagismo foi definido como hábito atual de fumar, independentemente do número de cigarros por dia e do tempo. O estado físico da paciente foi classificado segundo os critérios estabelecidos pela Sociedade Americana de Anestesiologia $(\mathrm{PI}-\mathrm{P} 6)^{14}$, sendo que neste estudo foram incluídas pacientes com estado físico até P3.

A técnica anestésica (bloqueio intercostal, bloqueio espinhal e anestesia geral) foi indicada no dia anterior, de acordo com a avaliação pré-anestésica e com o tipo de cirurgia. A duração do ato operatório foi definida como o tempo (minutos) compreendido entre o início (momento da incisão da pele) até o final do procedimento. Para a avaliação pós-anestésica, utilizou-se a escala de Aldrete modificadal', que atribui nota de 0 a 2 para cada um dos cinco parâmetros clínicos avaliados: consciência, atividade motora, respiração, circulação e saturação periférica de oxigênio. Considerou-se como critério para a alta da recuperação pós-anestésica a obtenção de pontuação máxima (10) neste índice, o que correspondeu à paciente: estar apta a mover as quatro extremidades; respirar profundamente e tossir; hemodinamicamente estável; totalmente desperta; saturação periférica de oxigênio acima de $92 \%$ em ar ambiente. O tempo (minutos) para que a paciente atingisse a pontuação máxima (dez) neste índice foi analisado.

Foram considerados efeitos adversos ${ }^{16,17}$ as intercorrências observadas no intra-operatório relacionadas aos procedimentos cirúrgico ou anestésico, no pós-operatório, na recuperação pós-anestésica e na unidade de internação. No intra-operatório, considerou-se sangramento anormal para a intervenção cirúrgica, assim como sangramento vaginal anormal no pós-operatório quando houve necessidade de intervenção específica. A dor foi considerada moderada ou intensa quando a paciente necessitou de medicação adicional à rotineiramente prescrita. A febre foi anotada como efeito adverso quando a temperatura axilar foi maior ou igual a $37,8^{\circ} \mathrm{C}$. Náuseas e vômitos, cefaléia e lipotimia foram avaliadas como presentes ou ausentes. No pós-operatório, foi anotado o momento em que ocorreu o efeito adverso e calculado o tempo decorrido entre 0 final do ato operatório e o diagnóstico da intercorrência. Para a análise, o tipo de cirurgia foi agrupado por região anatômica: torácica e pélvica.

A análise dos dados utilizou os testes Kruskall-Wallis e de Wilcoxon. A magnitude da associação das variáveis categóricas foi estimada por meio de valores de odds ratio, com respectivos intervalos de confiança a $95 \%$.

\section{Tabela I - Distribuição das mulheres de acordo com o tipo e topografia da intervenção cirúrgica e com a técnica anestésica}

\begin{tabular}{|c|c|c|}
\hline \multirow{2}{*}{$\begin{array}{l}\text { Tipo da intervenção cirúrgica } \\
\text { Pélvica }\end{array}$} & \multirow[t]{2}{*}{$\mathrm{n}$} & \multirow[t]{2}{*}{$\%$} \\
\hline & & \\
\hline Colouterino & 47 & 29,6 \\
\hline Vulva & 5 & 3,1 \\
\hline Laparoscopia & I & 0,6 \\
\hline Subtotal & 53 & 33,3 \\
\hline \multicolumn{3}{|l|}{ Torácica } \\
\hline Mama & 99 & 62,3 \\
\hline Inserção de port-a-cath & 7 & 4,4 \\
\hline Subtotal & 106 & 66,7 \\
\hline Total & 159 & 100 \\
\hline Técnica anestésica & $n$ & $\%$ \\
\hline \multicolumn{3}{|l|}{ Bloqueio espinhal } \\
\hline Raquianestesia & 41 & 25,8 \\
\hline Anestesia peridural & 10 & 6,3 \\
\hline Subtotal & 51 & 32,1 \\
\hline \multicolumn{3}{|l|}{ Bloqueio intercostal } \\
\hline Bloqueio intercostal Posterior único (BIPU) & 64 & 40,3 \\
\hline Bloqueio intercostal na linha Axilar média (BILAM) & 3 & 1,9 \\
\hline Subtotal & 67 & 42,1 \\
\hline Anestesia geral & 41 & 25,8 \\
\hline Total & 159 & 100 \\
\hline
\end{tabular}

\section{Resultados}

A média da idade das mulheres foi de 49, I anos, variando de 13 a 85 anos, com mediana de 48 anos. A média do índice de massa corpórea (IMC) foi de $27,2 \mathrm{~kg} / \mathrm{m}^{2}$, variando de 15,9 a $42,5 \mathrm{~kg} / \mathrm{m}^{2}$ e a mediana de $26,6 \mathrm{~kg} / \mathrm{m}^{2}$.

Em relação ao estado físico das pacientes, de acordo com os critérios estabelecidos pela Sociedade Americana de Anestesiologia (PI - P6), as pacientes foram distribuídas em PI (33,3\%), P2 (57,9\%) e P3 (8,8\%). A duração do ato operatório variou de 5 a 140 minutos, com média de 36,7 minutos e mediana de 35 minutos. Na região pélvica, as intervenções cirúrgicas no colo uterino foram as mais freqüentes (29,6\%); na região torácica, foram as mamárias (62,3\%) (Tabela I). As técnicas anestésicas mais utilizadas foram bloqueio intercostal posterior único (40,3\%), anestesia geral $(25,8 \%)$ e a raquianestesia $(25,8 \%)$ (Tabela I).

A média de tempo para obtenção de pontuação 10, no índice de recuperação pós-anestésica, foi de 84,9 minutos e mediana de 75 minutos (25 a 270 minutos). Nas mulheres que receberam anestesia geral, o tempo para alta da recuperação pós-anestésica (pontuação I0) foi significativamente maior $(p=0,0018)$ do que o observado para 0 bloqueio intercostal. Não houve diferença estatisticamente significante no tempo de recuperação pós-anestésica entre mulheres submetidas à anestesia geral versus bloqueio espinhal, e bloqueio intercostal versus bloqueio espinhal. O tempo para obtenção de pontuação 10 na escala 


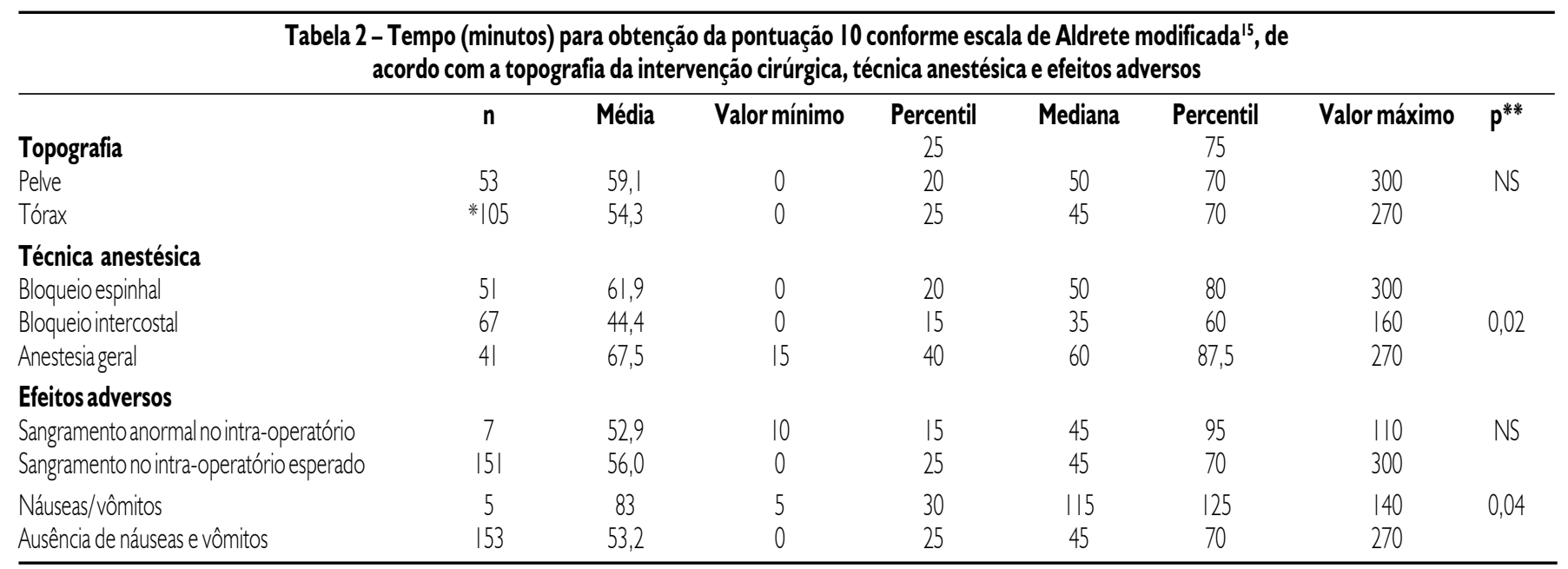

* Um caso ignorado por falha nas informações ***Teste de Kruskall Wallis

Wilcoxon of Two Sample Test (técnica anestésica): bloqueio espinhal versus bloqueio intercostal $(p=0,1528)$; bloqueio espinhal versus anestesia geral ( $p=0,1$ I 47$)$; bloqueio intercostal versus anestesia geral ( $p=0,00 \mid 8)$

de Aldrete modificada não foi estatisticamente diferente entre as mulheres submetidas a intervenções cirúrgicas na pelve e na região torácica (Tabela 2).

Em sete casos, observou-se sangramento anormal no período intra-operatório, sendo três intervenções cirúrgicas mamárias, uma inserção de port-a-cath e três intervenções cirúrgicas em colo uterino (conização). A análise dos dados mostrou não haver associação significativa deste efeito adverso com maior tempo de permanência na recuperação pós-anestésica. As mulheres que apresentaram náuseas e vômitos permaneceram por um tempo significativamente maior $(p=0,04)$ na recuperação pós-anestésica em relação àquelas que não apresentaram estes efeitos adversos (Tabela 2).

Os efeitos adversos mais freqüentes foram vômitos e náuseas, seguidos por dor moderada ou intensa. Destes efeitos, $58 \%$ foram observados nas primeiras quatro horas e $80 \%$ em até seis horas de pós-operatório. Nas mulheres submetidas a bloqueio espinhal, a incidência de náuseas e vômitos foi menos freqüente do que nas que receberam outras técnicas anestésicas $(O R=0,32 ;$ IC 95\% $=0,1$ I 0,87 ). Dor pós-operatória intensa ou moderada esteve mais freqüentemente associada ao bloqueio espinhal $(\mathrm{OR}=8,34$; IC $95 \%=1,90-41,82)$, às intervenções cirúrgicas realizadas na pelve $(\mathrm{OR}=5,22 ; \mathrm{IC} 95 \%=1,37-21,43)$ e ao hábito de fumar. Dentre as mulheres que apresentaram dor, $67 \%$ eram tabagistas, enquanto que dentre as que não referiram dor apenas $24 \%$ eram tabagistas (OR de 6,06 e IC de I,7I-2I,46) (dados não mostrados).

Dentre os 159 sujeitos da pesquisa, 64 apresentaram pelo menos um efeito adverso (Tabela 3). Todas as mulheres receberam alta 24 horas após a intervenção cirúrgica, e em apenas um caso foi observada uma complicação tardia, diagnosticada como cefaléia pós-raquianestesia, que necessitou de reinternação.

\section{Discussão}

Os resultados deste estudo mostraram que, em intervenções cirúrgicas ginecológicas e mamárias de pequeno porte, a ocorrência de algum efeito adverso é relativamente freqüente, sem, no entanto, apresentar relevância clínica. Os vômitos foram observados com maior frequêencia e constituem um impedimento para a alta hospitalar em intervenções cirúrgicas de pequeno porte realizadas em nível ambulatorial. Podem ocorrer precocemente e ser controlados com drogas antieméticas - usadas profilaticamente - embora não suficientemente efetivas, ou mesmo quando a paciente está nauseada ${ }^{18-22}$.

Uma revisão sistemática relatou que a proporção de pacientes que podem apresentar náuseas e vômitos depois de uma intervenção cirúrgica é variável e não necessariamente está associada com a técnica anestésica ${ }^{20}$. A associação de medicamentos diversos pode explicar diferentes resultados entre estudos publicados ${ }^{21}$. Neste estudo, náuseas e vômitos no pós-operatório foram menos freqüentes nas mulheres submetidas ao bloqueio espinhal do que naquelas que receberam anestesia geral ou bloqueio intercostal, o que pode ser atribuído ao emprego de anestésico local como agente único. A maior freqüência de vômitos em mulheres submetidas ao bloqueio intercostal e à anestesia geral pode dever-se ao emprego de opióides associados ao anestésico local e como droga auxiliar em anestesia geral23. Na anestesia geral, outros agentes como os voláteis - freqüentemente empregados na manutenção da anestesia - também têm efeito emetizante e no bloqueio espinhal pode ocorrer uma complicação como conseqüência da hipotensão arteria|20.

O bloqueio intercostal foi realizado nas mulheres que se submeteram à intervenção cirúrgica na mama; todavia não há evidências de que a presença de náuseas e vômitos esteja relacionada com a região topográfica destas intervenções cirúrgicas ${ }^{21}$. Outro fator que pode causar náuseas e vômitos é a tração de órgãos pela manipulação cirúrgica que provocaria reflexos parassimpáticos ${ }^{24}$, porém se aplicaria às intervenções cirúrgicas realizadas na pelve, como curetagem e conização.

Por outro lado, as mulheres submetidas ao bloqueio espinhal apresentaram mais freqüentemente dor do que aquelas submetidas à anestesia geral e bloqueio intercostal. Tal achado poderia ser explicado 
Tabela 3 - Distribuição das mulheres ( $n$ ) de acordo com os efeitos adversos e o momento de ocorrência (horas) após o término do procedimento

\begin{tabular}{|c|c|c|c|c|c|c|c|c|c|c|c|c|}
\hline \multirow[t]{2}{*}{ Efeitos adversos } & \multicolumn{9}{|c|}{ Tempo de ocorrência do primeiro efeito adverso (em horas) } & \multicolumn{3}{|c|}{ Mulheres que apresentaram efeitos adversos } \\
\hline & $0-1$ & $1-2$ & $2-3$ & $3-4$ & $4-5$ & $5-6$ & $6-7$ & $7-8$ & $>8$ & Total & Distribuiçãa & Incidência \\
\hline Vômitos e náuseas & 2 & 4 & 8 & 8 & 2 & 8 & । & । & 3 & 37 & $58 \%$ & $23,3 \%$ \\
\hline Dor & - & 3 & 2 & 2 & । & 2 & - & - & 3 & 13 & $20 \%$ & $8,2 \%$ \\
\hline Sangramento vaginal & - & 2 & 2 & । & - & - & । & - & । & 7 & $11 \%$ & $4,4 \%$ \\
\hline Cefaléia & - & I & - & - & - & - & - & - & । & 2 & $3 \%$ & $1,2 \%$ \\
\hline Lipotimia & - & I & - & I & I & - & - & - & - & 3 & $5 \%$ & $1,9 \%$ \\
\hline Febre & - & - & - & - & - & - & - & - & 2 & 2 & $3 \%$ & $1,2 \%$ \\
\hline Total & 2 & 11 & 12 & 12 & 4 & 10 & 2 & I & 10 & 64 & $100 \%$ & $40,2 \%$ \\
\hline Distribuição & $3 \%$ & $17 \%$ & $19 \%$ & $19 \%$ & $6 \%$ & $16 \%$ & $3 \%$ & $2 \%$ & $16 \%$ & & $100 \%$ & \\
\hline Distribuição acumulada & $3 \%$ & $20 \%$ & $39 \%$ & $58 \%$ & $64 \%$ & $80 \%$ & $83 \%$ & $84 \%$ & $100 \%$ & & & \\
\hline
\end{tabular}

pelo menor tempo de analgesia residual promovido pelo bloqueio espinhal, uma vez que neste foi utilizado anestésico local como agente único, sem associação com opióide, diferentemente da técnica adotada para o bloqueio intercostal. A associação de opióides aos anestésicos locais em anestesia regional, por melhorar a eficácia analgésica, reduz a necessidade do uso de opióides e outras drogas potencialmente emetizantes e, conseqüentemente, a ocorrência de efeitos colaterais como náuseas, vômitos, sedação intensa e depressão respiratória ${ }^{25}$. 0 emprego de protocolos para analgesia pós-operatória deve ser estimulado para reduzir a intensidade da dor, visando o conforto da paciente e minimizando a intensidade dos efeitos adversos e o tempo de permanência hospitalar ${ }^{25}$.

A febre ocorreu em I,2\% das mulheres, o que é concordante com outros autores que observaram que a freqüência deste efeito adverso durante o período pós-operatório é baixa, e parece estar na dependência da duração da intervenção cirúrgica, idade da paciente, preexistência de inflamação e local da intervenção cirúrgica, ${ }^{9}$. Neste estudo, apenas três mulheres apresentaram cefaléia, sendo que em duas delas pode ser atribuída a perfuração acidental da duramáter.

Os antecedentes de hipertensão arterial, diabetes melito, cardiopatia, IMC e estado físico não influenciaram no tempo de recuperação anestésica, na ocorrência e no tempo para o aparecimento de efeitos adversos. A dor no período pós-operatório foi mais freqüente nas mulheres tabagistas, fato descrito anteriormente por outros autores, sendo esta manifestação clínica também relacionada ao grau de ansiedade ${ }^{26}$.

Embora o estudo tenha sido realizado em hospital universitário com participaçãa de diferentes equipes anestésicas e cirúrgicas, não há dados que permitam inferir que este aspecto tenha interferido na ocorrência de efeitos adversos.

Atualmente admite-se como novo paradigma para intervenções cirúrgicas ambulatoriais a transferência de pacientes diretamente da sala de cirurgia para uma unidade de recuperação (fast tracking), sem passar pela sala de recuperação anestésica, com o objetivo de reduzir a permanência do paciente no centro cirúrgico e a otimização dos recursos físicos com a necessária segurança. $O$ sucesso desta sistemática de trabalho depende de ajustes apropriados da técnica anestésica para permitir a recuperação mais rápida do paciente e a prevenção dos efeitos adversos mais comuns, tais como dor, náuseas e vômitos ${ }^{27}$.

Os resultados deste estudo servem de subsídio para orientar a escolha de técnicas anestésicas adequadas para a realização deste tipo de procedimento, visando minimizar a ocorrência de efeitos adversos que possam prolongar o tempo de internação. As mulheres que se submeteram ao bloqueio intercostal obtiveram mais rapidamente pontuação 10 e, portanto, alta mais precoce na recuperação pósanestésica avaliada pelo índice de Aldrete e Kroulik ${ }^{15}$. Esta técnica proporcionou anestesia adequada, rápida recuperação, menor incidência de efeitos adversos e boa aceitação da paciente, constituindo vantagens para a mulher, para o hospital e para a comunidade ${ }^{28,29}$. Com isso, espera-se a diminuição do custo hospitalar, liberação de leitos para casos de maior necessidade, menor ansiedade ocasionada pelo menor tempo de internação e menor risco de infecção hospitalar.

\section{Conclusão}

O período de seis horas de observação mostrou-se adequado para a avaliação da maioria das complicações e efeitos adversos que ocorrem após intervenções cirúrgicas, ginecológicas e mamárias, de pequeno porte. A dor no período pós-operatório é mais freqüente em mulheres submetidas ao bloqueio espinhal e com antecedentes de tabagismo.

\section{Conflito de interesse: não há}

\section{SUMMARY}

AdVerse postoperative efFects in minor gynaecological

\section{AND BREAST SURGERIES}

OBIECTNES. To analyze the occurrence of adverse effects in the first 24 hour postoperative/postanesthetic period in women undergoing minor gynecologicorminor breastsurgeries and to identify main associated factors.

METHODS. A cross-sectionalstudy was conducted with 159 women who underwentminorgynecologicorbreastsurgeries. The womenwereadmitted to the hospital one day before surgery and remained hospitalized for at least 24 hours aftersurgery. The anesthetictechniques performed were intercostal nerve blockade, spinal anesthesia, and general anesthesia.

RESULTS. Tthe mostfrequentadverse effects were vomiting, nausea and 
pain thatoccurred in $40.3 \%$ of women. Ofthese effects, $60 \%$ were observed in the firstfour hours and $80 \%$ were observed in up to six hours after surgical intervention. Women submitted to intercostal blockade received earlier postanesthetic release. Spinal anesthesia was most frequently associated with postoperative pain, although with a lower incidence of nausea and vomiting when compared to generalanesthesia and intercostalblockade. The incidence of pain was higher in women who smoked.

CONCLUSIONS. Asix-hourperiod of postoperative observationappeared to be adequate for assessment of most complications and adverse effects occurring in women who undergo minorgynecologicorminorbreastsurgeries. [Rev Assoc Med Bras 2006; 52(4): 203-7]

KEY wORDs: Ambulatory surgical procedures. Gynecology. Breast. Anesthesia.

\section{RefERÊNCIAS}

I. Yamashita AM. Anestesia ambulatorial. In: Yamashita AM, Takaoka F, Auler Jr JOC, Iwata NM. Anestesiologia: SAESP. São Paulo: Atheneu; 2001. p.997-1013.

2. Schmidt A, Bagatini A. Náusea e vômito pós-operatório: fisiopatologia, profilaxia e tratamento. Rev Bras Anestesiol 1997;47:326-34.

3. Williams BA, Vogt MT, Kentor ML, Figallo CM, Kelly MD, Williams JP. Nausea and vomiting after outpatient $A C L$ reconstruction with regional anesthesia: are lumbar plexus blocks a risk factor? J Clin Anesth 2004; |6:276-8|.

4. Reurer M, Hueppe M, Klotz KF, Beckhoff M, Hennig J, Netter P, et al. Detection of causal relationships between factors influencing adverse sideeffects from anaesthesia and convalescence following surgery: a path analytical approach. Eur J Anaesthesiol 2004;2:434-42.

5. Habib AS, White WD, Eubanks S, Pappas TN, Gan TJ. A randomized comparison of a multimodal management strategy versus combination antiemetics for the prevention of postoperative nausea and vomiting. Anesth Analg 2004:99:77-81.

6. Hines R, Barash PG, Watrous G, O'Connor T. Complications occurring in the postanesthesia care unit: a survey. Anesth Analg 1992;74:503-9.

7. Varaklis K, Stubblefield PG. Evaluating the role of incidental diagnostic dilation and curettage in young women undergoing elective laparoscopic sterilization. J Reprod Med 1995;40:4I 5-7.

8. Vaghadia H, Scheepers L, Merrick PM. Readmission for bleeding after outpatient surgery. Can J Anaesth 1998;45: 1079-83.

9. Frank SM, Kluger MJ, Kunkel SL. Elevated thermostatic setpoint in postoperative patients. Anesthesiology 2000;93: | 426-31.

10. Thong WY, Strickler AG, Li S, Stewart EE, Collier CL, Vaughn WK, et al. Hyperthermia in the forty-eight hours after cardiopulmonary bypass. Anesth Analg 2002;95: I 489-95.

I I. Chiharu N, Lenhard TR. Fever during anaesthesia. Best Practice Res Clin Anaesth 2003; 17:499-5 17.
12. Gold BS, Kitz DS, LeckyJH, Neuhaus JM. Unanticipated admission to the hospital following ambulatory surgery. JAMA 1989;262:3008-10.

13. Chung F, Mezei G. Intraoperative adverse events during ambulatory surgical procedures. Can J Anesth 1992;39:440-8.

14. ASA. American Society of Anasthesiologists. Physical Status Classification System. 2006 [on line]. Avaliable from: http://www.asahq.org/clinical / physicalstatus.htm. [cited 2006 jan I I]

I5. Aldrete JA. The post-anesthesia recovery score revisited. J Clin Anesth |995:7:89-9|.

16. Descritores em ciência da saúde: DeCS. Biblioteca Virtual em Saúde. Efeitos adversos [on line] Disponível em:http://decs.bvs.br/cgi_bin/ wxis. 1660. [citado 2004 jan]

17. Udelsmann A. Complicações anestésicas. In: Yamashita AM, Takaoka F, Auler Jr JOC, Iwata NM. Anestesiologia: SAESP. São Paulo: Atheneu; 200I.p. 1029-55.

18. Watcha MF, White PF. Postoperative nausea and vomiting. Its etiology, treatment, and prevention. Anesthesiology 1992;77: 162-84.

19. Resolução CFM no 1.409/94. Normas para atos cirúrgicos ou endoscópicos em regime ambulatorial. Diário Oficial da União, Brasília (DF); 1994 | 4 jun

20. McQuay HJ, Moore RA. Postoperative analgesia and vomiting, with special reference to day-case surgery: a systematic review. Health Technol Assess 1998;2:1-236.

21. Liu SS, Strodtbeck WM, Richman JM, Wu CL. A comparison of regional versus general anesthesia for ambulatory anesthesia: a meta-analysis of randomized controlled trials. Anesth Analg 2005; I 0 I : 1 634-42.

22. Kokinsky E, Nilsson K, Larsson LE. Increased incidence of postoperative nausea and vomiting without additional analgesic effects when a low dose of intravenous fentanyl is combined with a caudal block. Paediatr Anaesth 2003; | 3:334-8.

23. Hamaji M, Oka N, Tashiro C, Seki T, Miyata M, Kawashima Y. Anaesthetic management with morphine in phaeochromocytoma. Can Anaesth Soc J 1984:31:681-6.

24. Bonnet $F$, Marret $E$. Influence of anaesthetic and analgesic techniques on outcome after surgery. Br J Anaesth 2005;95:52-8.

25. Chung F, Ritchie E, Su J. Postoperative pain in ambulatory surgery. Anesth Analg 1997;85:808-16.

26. Murauski JD, Gonzalez KR. Peripheral nerve blocks for postoperative analgesia. AORN J 2002;75: I 36-47.

27. Yunus MB, Arslan S, Aldag JC. Relationship between fibromyalgia features and smoking. Scand J Rheumatol 2002;31:30 I-5.

28. Joshi GP, Twersky RS. Fast tracking in ambulatory surgery. Ambul Surg 2000;8: 185-190

29. Hadzic A, Arliss J, Kerimoglu B, Karaca PE, Yufa M, Claudio RE, et al. A comparison of infraclavicular nerve block versus general anesthesia for hand and wrist day-case surgeries. Anesthesiology 2004; 10 | : | 27-32.

Artigo recebido: 27/01/05

Aceito para publicação: 07/06/06 